\title{
9. OBSERVATIONAL ASPECTS OF GALACTIC MAGNETIC FIELDS
}

Introductory Report

(Friday, September 12, 1969)

G. L. VERSCHUUR

National Radio Astronomy Observatory*, Green Bank, W.Va., U.S.A.

\section{Introduction}

I will review the impressive advances in the observations of the galactic magnetic field made since the time of van de Hulst's review at the 1966 Noordwijk Symposium (van de Hulst, 1967). Most of these observations are so recent that the consequences have not yet been worked out very well and are in need of discussion. Luckily the emphasis in the present Symposium, unlike that in many others, is on discussion. For this reason I will not hesitate to include in my review provocative speculations. I will base my discussion on Mathewson's (1968) elegant magnetic field model, consisting of a local field in the form of a sheared helix, superimposed on a large scale longitudinal field. I will include the criticism of this model by Gardner et al. (1969b). Mathewson has succeeded in accounting for such data as the distribution of background polarized radiation from the Galaxy, the distribution of rotation measures of extragalactic radio sources and even the spurs and ridges in galactic continuum emission. There are critics who inherently distrust models that account for too many things at one time, but I feel that we should try to account for as many things as possible with the least number of models. Mathewson has succeeded in uniting much data and I will only add a few pieces to his model.

The progress since 1966 in the number, variety, and quality of the observations is indeed impressive. Gardner et al. (1969a) have presented data on the radio polarization of 366 sources at three frequencies. The observations have been discussed by Gardner and Whiteoak (1969) and Gardner et al. (1969b). Mathewson has largely filled in the gap in optical polarization data in the southern hemisphere by measurements on 2000 stars, so that his model could be based on 7000 stars in the northern and southern skies together. Enormous progress has also been made in the area of direct measurements of the field strength. First, measurements of the Zeeman splitting at $21 \mathrm{~cm}$ has shown the existence of fields of a few $\mu \mathrm{G}$ up to $50 \mu \mathrm{G}$. Second, the discovery of pulsars has made it possible to measure both the (Faraday) rotation measure (RM) and the signal-dispersion measure (DM); the ratio of these two yields directly an estimate of the mean value of the line-of-sight component of the magnetic field. These results give average field strengths between a few times $0.1 \mu \mathrm{G}$ to $3 \mu \mathrm{G}$.

I start the review with Table I which is my adaptation of a table originally presented

* Operated by Associated Universities, Inc., under contract with the National Science Foundation. 
TABLE I

Observational data about the galactic magnetic field (modified from the original version by Van de Hulst, 1967)

Category

A

Optical interstellar polarization

Polarization of non-thermal radio emission

Faraday effect on radio sources

B Elongation of interstellar clouds

C Cosmic-ray energy, density and confinement

D Cosmic-ray anisotropy

E Zeeman effect, $\mathrm{H}$

F Zeeman effect, $\mathrm{OH}$

G Pulsars

Magnitude

Direction

Key: $\mathrm{e}=$ excellent $\mathrm{f}=$ fair or fine; $\mathrm{q}=$ questionable or marginal $;-=$ no data, or do not believe.

by van de Hulst (1967). The present table (which makes clear what my biases are) groups several fields of research together in a more optimistic way than the analogous display of 1966. One should appreciate the amount of data included in category A which encompasses the results of radio-source polarization measurements on hundreds of sources, continuum surveys, background polarization data, and optical polarizations for thousands of stars. Category B includes the shapes of filamentary nebulae (following a proposal by Shajn, 1955). Van de Hulst left this category out, but, as we will explain in Section 7, we disagree with him on this point.

\section{Mathewson's Model and Associated Work}

Mathewson (1968) has combined his southern polarization data on 1400 stars (mostly within $500 \mathrm{pc}$ of the Sun) with the northern hemisphere results (a total of 7000 stars), in order to deduce a magnetic field model for the solar neighborhood. Figure la shows the polarization data, as projected on the sky. As is well known, the polarization vector gives the average of the direction of the magnetic field component perpendicular to the line of sight. Mathewson then took a helical field structure, and with a trial-anderror method he chose the parameters of this helix such that they predicted maps (those of Figures $1 \mathrm{~b}$ and $1 \mathrm{c}$ ) as similar as possible to Figure la. The best-fitting model has the field lines form tightly wound (pitch angle $7^{\circ}$ ), right-hand helices which lie on the surfaces of tubes having elliptical cross-sections of axial ratio 3 with major axes parallel to the galactic plane. The helical pattern is sheared by $40^{\circ}$ on the plane of the Galaxy, in an anti-clockwise sense when viewed from the galactic North Pole. The Sun is $100 \mathrm{pc}$ toward the galactic center from the magnetic axis, and 10 pc below the galactic plane. Two predicted maps are shown in Figures $1 \mathrm{~b}$ and $1 \mathrm{c}$. Figure $1 \mathrm{~b}$ shows the component, perpendicular to the line of sight, of the tangents to helices with a semi-major axis of $250 \mathrm{pc}$, and Figure 1c represents in the same way the families of helices with semi-major axes of $115 \mathrm{pc}$ (thick lines) and $100 \mathrm{pc}$ (thin lines). The differ- 
ences between Figures $1 \mathrm{~b}$ and $1 \mathrm{c}$ are due mainly to the offset in the Sun's position with respect to the axes of the helices. It is seen that the superposition of Figures $1 \mathrm{~b}$ and $1 \mathrm{c}$ very well duplicate Figure 1a. Already a first look at Figure la shows that the field lines are curved. The picture does not seem to agree at all with parallel lines converging in the distance. This disagreement, it seems, is the basis for Mathewson's assertion that the observations are not compatible with a longitudinal field. I therefore differ in opinion from Gardner et al. (1969b) who claimed that the data shown in Figure
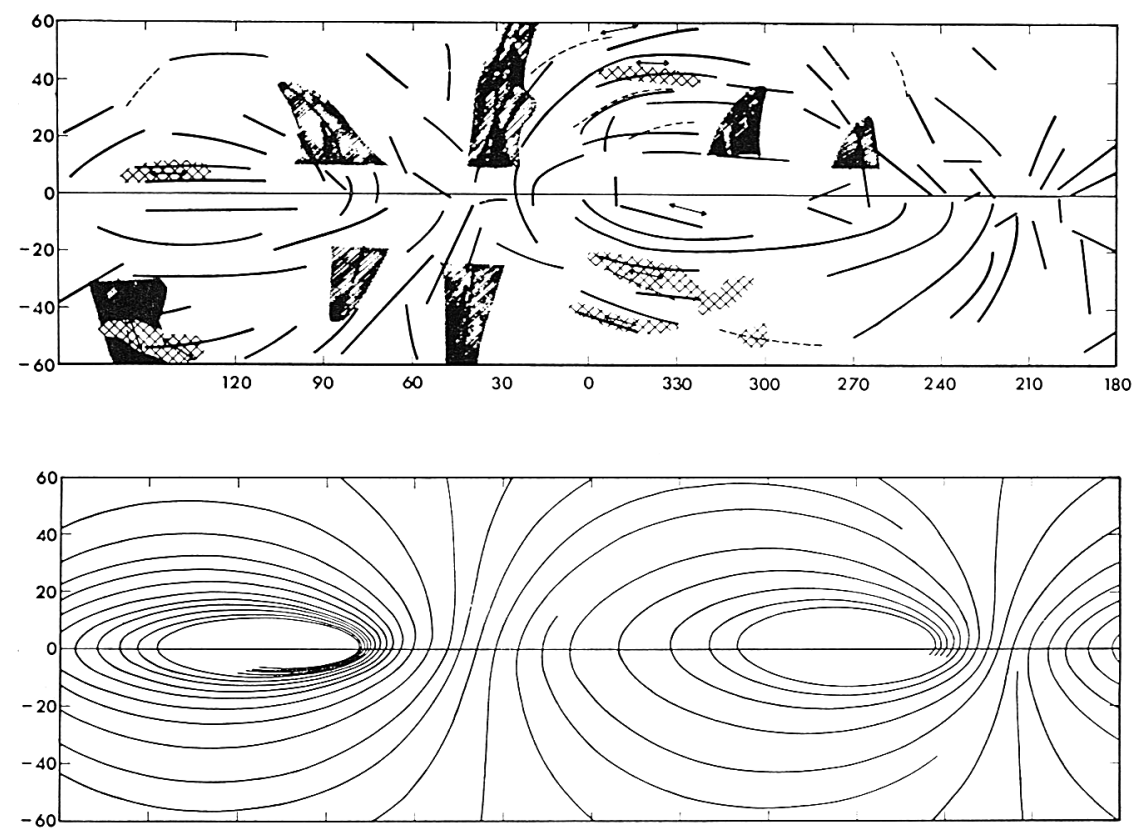

$(b)$

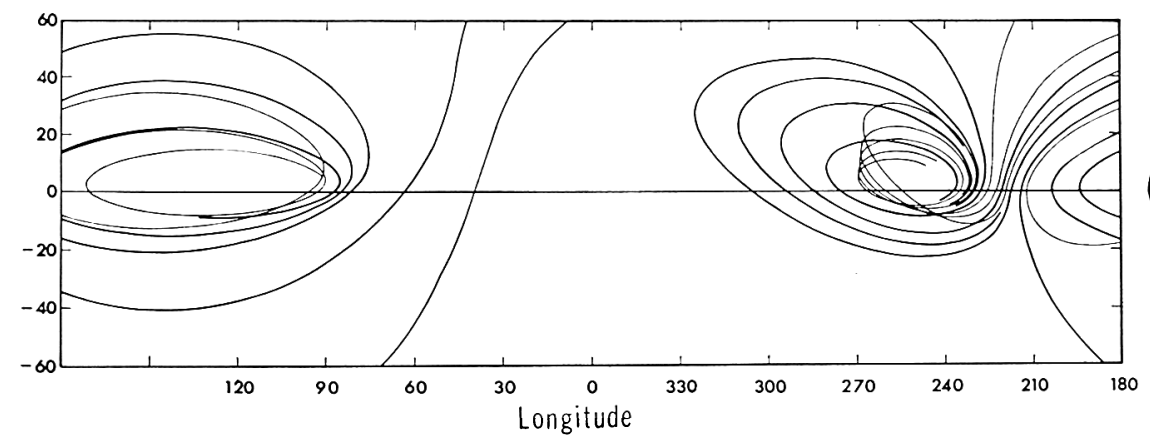

Fig. 1. Optical polarization data and field models from Mathewson (1968). - (a) Heavy lines represent 'flow patterns' formed by $E$-vectors of optical polarization. Radio spurs are shaded. Crosshatched areas are strongly polarized at $408 \mathrm{MHz}$. Arrowed lines give directions of magnetic field obtained from radio measurements. - (b, c) Projection on sky of several families of helices (see text). 
$1 \mathrm{a}$ "... are also consistent with an axial field toward $l^{\mathrm{II}}=50^{\circ}$ ". Still, such a field would have been indicated if a smaller sample of stars had been taken, especially if the stars were near the plane. Therefore I do not give much weight to Seymour's (1969) analysis of 550 stars which yields an axial field. It would be of interest if Seymour were to apply his analyzing method to all the polarization data now available.

Also included in Mathewson's 'Unified Field Theory' are data by Appenzeller (1968) on 308 stars near the poles and (in the plane) near the anomalous region around $l^{\mathrm{II}}=140^{\circ}$. Gardner, Morris, and Whiteoak claim that “... Appenzeller found a low altitude field aligned parallel to Gould's belt in Perseus $\left(l^{\mathrm{II}}=145^{\circ}\right)$ " and then state "this orientation defies explanation in terms of the magnetic helix". These are strong words, first since Appenzeller's data do fit Mathewson's model, in particular in the North Pole region (these data are not shown in Figure 1 but may be found in Mathewson, 1968); and second since Mathewson (1969) himself had already stated that the helical field is probably associated with Gould's belt. Appenzeller does not, in so many words, invoke Gould's belt and admits that more data are needed in any case before a comprehensive model can be made. Much needs to be said on the association of the magnetic field structure and the distribution of stars in the Gould belt system, but I should perhaps leave that for the discussion. Clube (1968) has been rather specific on this point but he unfortunately used the contour map of Gardner and Davies (1966) which has been superseded by later work.

Before I move to the modification of the helical model I would like to stress the impressive property of this model in that it provides a simple explanation for the spurs and ridges of continuum radio emission which may be tracers of the field lines. Non-thermal electrons are injected into the helix, probably from sources nearer the plane, and these electrons then spiral away from the plane along the field lines and appear as enhanced spurs of emission. Bingham (1967) had already shown that field lines do indeed lie along the galactic spurs. Hall (1958) had noted that vectors from $l^{\mathrm{II}}=350^{\circ}$ to $40^{\circ}$ seemed to converge to $l^{\mathrm{II}}=35^{\circ}$ and he speculated that this might have a 'particular significance'. This point is at the base of the North Polar spur and Figure 1 shows what the significance is.

In a more recent paper Mathewson and Nicholls (1968) propose a modification of the helical model in order to account for Faraday rotation data. Consider first the contribution by the local helical field. As is known, the Faraday rotation measure (RM) equals $0.81 \int n_{e} B_{\|} \mathrm{d} l \mathrm{rad} \mathrm{m}^{-2}$ (if $n_{e}$ is in $\mathrm{cm}^{-3}, l$ in pc, and $B_{\|}$in $\mu \mathrm{G}$ ), where $l$ is the distance along the line of sight. Because of the condition $\operatorname{div} \mathbf{B}=0$, the geometry determines the field strength along the field lines in terms of the strength at, say, the minor axis of the elliptical cross sections of the helix. This makes it possible to construct contour maps of $B_{\|}$, one of which is shown in Figure 2. The contours are in units of 0.025 times the field strength on the minor axis of the helical cross section. The semi-major axis is $250 \mathrm{pc}$, but any helix wound around a tube of larger cross section would give a similar pattern; smaller tubes would not, because the Sun is off the axis of the family of tubes. (Note the difference between this Figure 2 and Figures $1 \mathrm{~b}$ and $1 \mathrm{c}$ : in the two latter figures the curves indicate only the direction of the field 


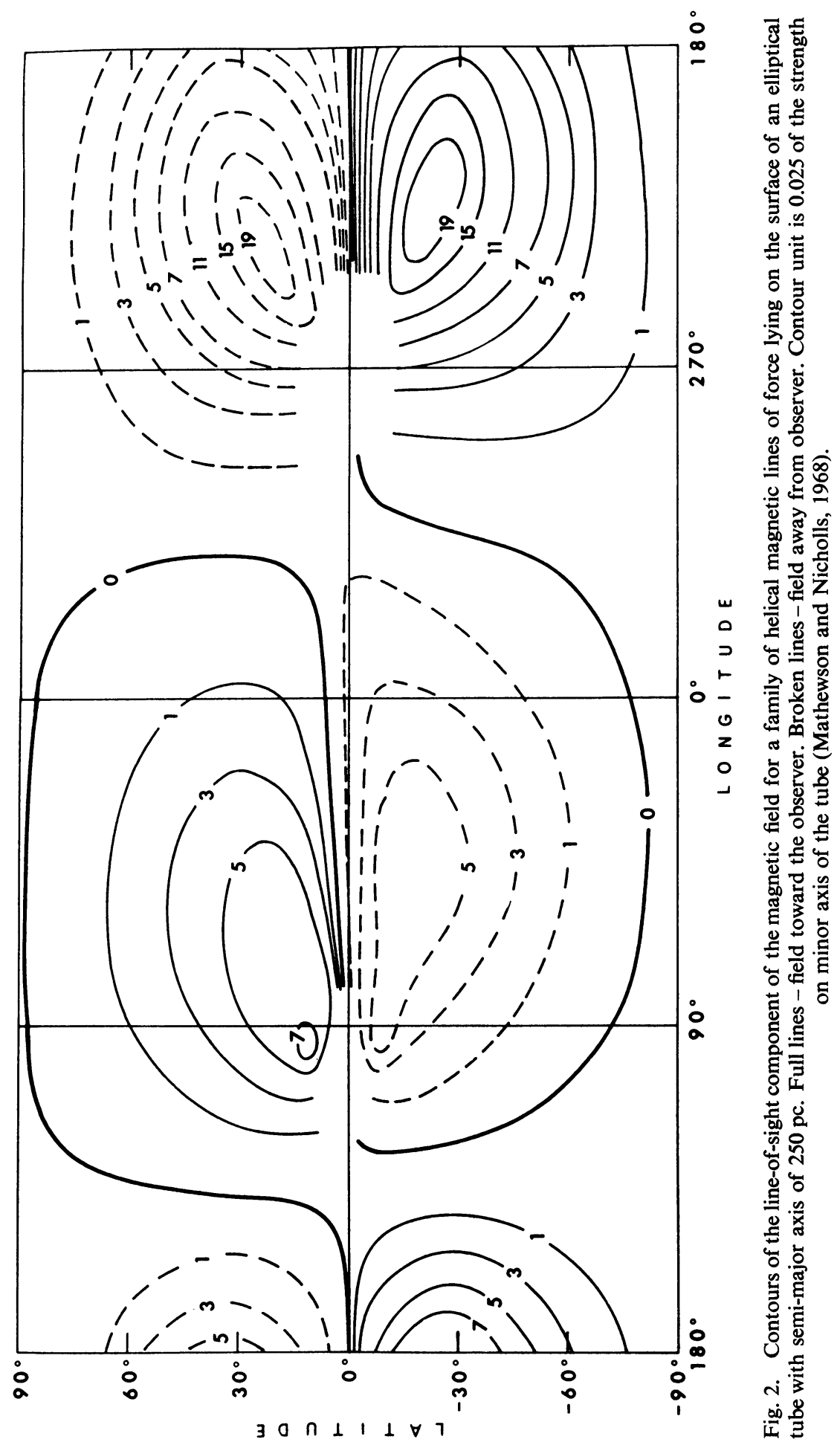


component perpendicular to the line of sight, i.e., Figures $1 \mathrm{~b}$ and $1 \mathrm{c}$ are not contour diagrams.) Mathewson and Nicholls (1968) claim that one can account for the RM distribution as found by Gardner et al. (1969a) by adding to the helical field a largerscale longitudinal field in the direction $l^{\mathrm{II}}=90^{\circ}$ and $b^{\mathrm{II}}=0^{\circ}$, i.e., the helix is just a superimposed local phenomenon.

I have a few critical remarks regarding this modified model. First, since the longitudinal field only exists beyond several hundred pc, one wonders how it can contribute to the rotation measure at high latitudes where one expects to find very few electrons. Second, Mathewson and Nicholls suggest that south of the galactic plane the longitudinal field dominates between $l^{\mathrm{II}}=320^{\circ}$ to $0^{\circ}$ and $l^{\mathrm{II}}=130^{\circ}$ to $160^{\circ}$, whereas in between these longitudes the combination of the longitudinal field, directed to $l^{1 \mathrm{I}}=90^{\circ}$, and the helical field (Figure 2) accounts for the RM-signs. But north of the plane it has to be the helical field that determines the RM-sign, either by virtue of its strength or as a result of higher local electron density. Since the pulsar data do not suggest a systematic difference in integrated electron content between the northern and southern hemispheres, there must be a stronger field present in the north. This of course destroys the symmetry in the model and takes away some of its elegance. In Section 6 I will return to the difficulties associated with the RM-distribution, but first I consider in the following sections the suggestion that the Galaxy has a basic longitudinal field, directed along the spiral arms and distorted by local structures which we will call 'field pockets'. I will show that, observationally at least, there is evidence for motion and structure of interstellar clouds which can be associated with our local field pocket.

\section{The Distance at Which Optical Polarization is Produced}

To test whether the helical field is indeed a local phenomenon we may investigate whether there is a correlation between the polarization of a star and its distance. Behr (1959) initially found that the polarization increased with distance but neither Hall (1958) nor Hiltner (1956) found such an effect. One pertinent observation indicating that most of the observed polarization is produced rather nearby is that by Krzeminski and Serkowski (1967). They noted that two clusters in the same area in the sky (around $l^{I I}=134^{\circ}$ ) had about the same degree of polarization, although their distances differed by large amounts, the first (Stock 2) being at 300 pc, the second (h and $\chi$ Persei) at 2000 to 3000 pc. Therefore, they concluded that probably most of the net observed polarization takes place in the solar neighborhood.

I have studied this problem using the large amount of data available on polarization of (open) clusters and associations. By taking groups of stars instead of individual field stars we hopefully reduce the 'noise' in the polarization data and in the distance determinations. One may study the magnitude of the polarization, the mean position angle and the scattering around this mean, all as a function of distance and as a function of $l$ and $b$. I collected data on 56 groups of stars containing five or more members with polarization data available (see Table II). I will not give details here but hope to publish these elsewhere. In Figure $3 a$ the mean polarization is plotted as a 
TABLE II

Mean polarization as function of distance for clusters and associations

\begin{tabular}{lll} 
Longitude range & Number of objects & Correlation coefficient \\
\hline $0^{\circ}-30^{\circ}$ & 10 & 0.25 \\
$60^{\circ}-89^{\circ}$ & 10 & -0.12 \\
$90^{\circ}-121^{\circ}$ & 11 & 0.38 \\
$122^{\circ}-180^{\circ}$ & 14 & 0.60 \\
$181^{\circ}-359^{\circ}$ & 11 & 0.39
\end{tabular}
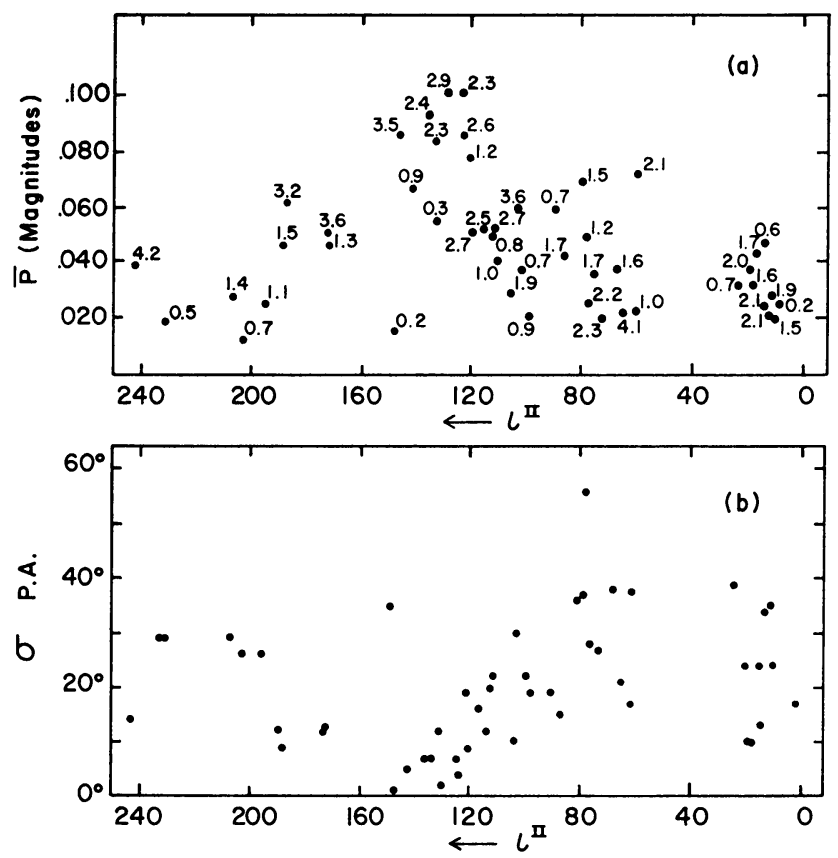

Fig. 3. Polarization properties of clusters and associations. - (a) Mean polarization as a function of longitude. Numbers indicate the distance to the cluster of association in kpc. - (b) The scatter in position angle of member stars (one standard deviation) as a function of longitude.

function of $l$ and $b$ with the distance to each cluster indicated next to the various points. It is clear that the effect noted by Krzeminski and Serkowski is evident elsewhere. Take, for instance, the group of objects near longitude $15^{\circ}$. The mean polarizations are all of about the same value, whereas their distances vary from 0.6 to 2.1 $\mathrm{kpc}$. To test this further, I estimated the correlation coefficient between mean polarization and distance in various longitude intervals. The results in Table II show that polarization is not a function of distance.

The scatter in polarization position angle for stars within the individual clusters and associations is shown as a function of longitude in Figure $3 \mathrm{~b}$ ( $\sigma$ equals one standard deviation value). The minimum scatter is around $l^{l \mathrm{II}}=140^{\circ}$ which is consis- 
tent with the helical model. Figure $3 \mathrm{~b}$ looks similar to a plot given by Hiltner (1956). The combination of Figures $3 a$ and $3 b$ is not inconsistent with the suggestion that the polarization is produced relatively locally, i.e., within about $500 \mathrm{pc}$ of the Sun.

\section{The Zeeman Effect}

The Zeeman splitting of the $21-\mathrm{cm}$ line has now been observed in a considerable number of neutral hydrogen clouds (Verschuur, 1968, 1969a, b, c; Davies et al., 1968). It often remains difficult to estimate the magnetic field strength due to the complexity of most of the spectra. It is interesting that in all the Zeeman observations made so far the directions of the fields fit Mathewson's helical-plus-longitudinal field model. There are, however, large problems with the field strengths. For example in Orion A $\left(l^{I I}=209, b^{I I}=-19^{\circ}\right)$ I find a dense cloud with a field of $-50 \mu \mathrm{G}^{*}$, whereas at the same longitude, but at the other side of the plane I was able to make one successful Zeeman observation (at $l^{\mathrm{II}}=238.25, b^{\mathrm{II}}=+38.05$ ), which yields a magnetic field of $4.5 \pm 5.3 \mu \mathrm{G}$. These values cannot be reconciled with the predictions of Figure 2 ; addition of a longitudinal field directed to $l^{\mathrm{II}}=90^{\circ}$ would make the disagreement even worse.

A twofold explanation for this seems to be, first, that the strongest fields are found in the densest clouds, which are gravitationally stable at least against internal motions, and second that the field strength is the result of amplification by contraction of the clouds (Verschuur, 1969d).** More data recently obtained (Verschuur 1969b, c) allow us to examine this suggestion further.

Table III lists the neutral hydrogen features in which fields have been detected, whereas Table IV contains information on a number of negative results. Column 4 gives the velocity of the feature with respect to the local standard of rest (1.s.r.); column 5, the field strength, from Verschuur (1969a, b, c); and column 6 gives the density in the clouds. These have either been taken from previously published works, listed in column 7, or estimated here. Column 8 indicates the reliability of the density estimates and (in some cases) of the field estimate. 'Good' indicates that little further improvement can be expected on the data given. 'Fair' indicates that further observations might help to improve the estimates, whereas 'Poor' implies that further observations are essential to establish the best values for these features.

For M17 I have measured an accurate absorption line profile and have used the observed dispersion $(\sigma)$ of the apparent Zeeman pattern, together with an estimate of the optical depth, to derive $n_{\mathrm{H}}$. This value differs a little from that suggested by Clark (1965). In general in Table III I have assumed a cloud diameter of $10 \mathrm{pc}$ whenever Clark did not give interferometer data. The data of Table III are shown in Figure 4, where the values of $B_{\|}($in $\mu \mathrm{G})$ are plotted against $n_{\mathrm{H}}\left(\right.$ in $\mathrm{cm}^{-3}$ ). The solid

* I have adopted the convention to call a value of $B_{\|}$negative if the field is directed toward the observer. This is in agreement with the generally-accepted definition of negative radial velocities. It is not in agreement with the less generally used definition of rotation measure.

** See also the remarks by Mestel during the following discussion, p. 187 (Ed.). 
error bars indicate well-determined field values and the dashed lines indicate less certain values or cases where only estimates of the field or density could be made.

For an isotropically contracting cloud with a 'frozen-in' magnetic field we expect the density to be $\propto R^{-3}$ ( $R=$ radius) whereas the magnetic field will be $\propto R^{-2}$. Therefore the field, $B$, will be proportional to $n_{H}^{2 / 3}$. I have drawn in Figure 4 a line

TABLE III

Magnetic fields in clouds of known density

\begin{tabular}{|c|c|c|c|c|c|c|c|c|}
\hline Direction & $l^{\mathrm{II}}$ & $b^{\text {II }}$ & $\begin{array}{l}\text { Velocity } \\
\left(\mathrm{km} \mathrm{sec}^{-1}\right)\end{array}$ & $\begin{array}{l}\text { Field estimate } \\
(\mu \mathrm{G})^{\mathrm{a}}\end{array}$ & $n_{\mathrm{H}}\left(\mathrm{cm}^{-3}\right)$ & References & $\begin{array}{l}\text { Integration } \\
\text { time (hr) }\end{array}$ & $\begin{array}{l}\text { Signi- } \\
\text { ficance }\end{array}$ \\
\hline \multirow[t]{2}{*}{ Tau A } & 185 & -6 & +10 & $-3.5 \pm 0.7$ & $14(16)$ & $1(2)$ & 75 & G \\
\hline & & & +4 & $-1.5 \pm 0.9$ & 27 & & & $\mathbf{F}$ \\
\hline \multirow{2}{*}{ Cas A } & 112 & -2 & -38 & $+18.0 \pm 1.9$ & 193 & 1 & 34 & G \\
\hline & & & -48 & $+10.8 \pm 1.7$ & 87 & 1 & & G \\
\hline \multirow[t]{2}{*}{ Cyg A } & 76 & +6 & +3 & $+3.0 \pm 2.2$ & $1-4$ & 3 & 26 & $\mathbf{F}$ \\
\hline & & & -84 & $+4.0 \pm 2.2$ & 2.5 & 3 & & F \\
\hline M17 & 15 & -1 & +14 & $+25.0 \pm 10.0$ & $60-100$ & $\begin{array}{l}\text { Estimate } \\
\text { (see text) }\end{array}$ & 9 & $\mathbf{P}$ \\
\hline \multirow[t]{2}{*}{ Orion $\mathbf{A}$} & 209 & -19 & +7 & $-50.0 \pm 15.0$ & 680 & 2 & 8 & G \\
\hline & & & +2 & $-70.0 \pm 20.0$ & 350 & 2 & & $\mathbf{P}$ \\
\hline
\end{tabular}

1. Verschuur, 1969a.

2. Clark, 1965.

3. Shuter and Verschuur, 1964.

a A negative sign indicates a field toward the observer.

b $\mathbf{G}=$ Good, $\mathbf{F}=$ Fair, $\mathbf{P}=$ Poor. See text.

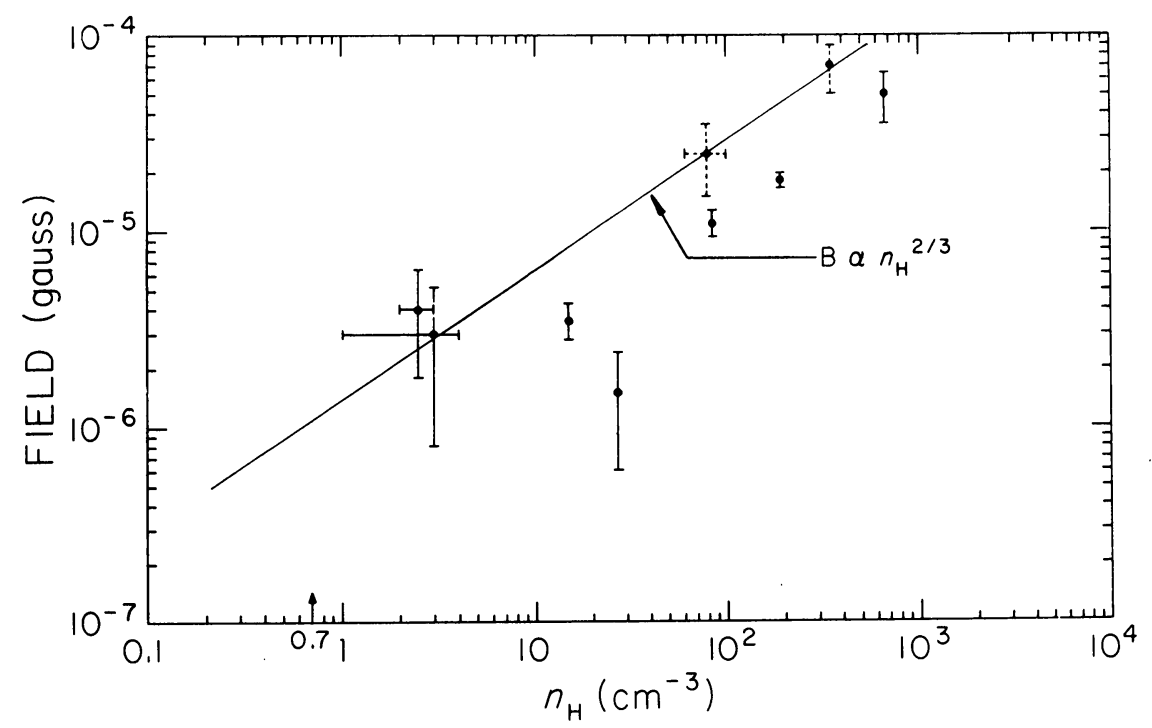

Fig. 4. Magnetic fields $(B)$ in neutral hydrogen clouds as a function of their density $\left(n_{\mathrm{H}}\right)$. The solid lines indicate well-determined values. The broken bars indicate less certain values. The line with slope $\frac{2}{3}$ shows the expected form for the case of magnetic fields frozen into contracting clouds. 
with a slope of $\frac{2}{3}$. It is clear that the line represents the observations very well. This suggests strongly that contracting clouds of neutral hydrogen do in fact carry 'frozenin' fields with them, without dissipation, when they contract over nearly three orders of magnitude in density.

Since one is only seeing one component of the field in the Zeeman data the upper envelope of the points in Figure 4 should describe the history of the field strength in a contracting cloud. If this argument is valid one can estimate the value at the onset of cloud formation by extrapolating back to the average interstallar hydrogen

TABLE IV

Magnetic fields in other clouds

\begin{tabular}{lllll}
\hline$l^{\text {II }}$ & $b^{\text {II }}$ & $\begin{array}{l}\text { Velocity } \\
\left(\mathrm{km} \mathrm{sec}^{-1}\right)\end{array}$ & $\begin{array}{l}\text { Field estimate } \\
(\mu \mathrm{G})^{\mathrm{a}}\end{array}$ & Integration time (hr) \\
\hline
\end{tabular}

$\begin{array}{lrrrrc}\text { Sag A } & 0 & 0 & -53 & -8.0 \pm 8.1 & 6 \\ \text { Sag A } & 0 & 0 & 0 & +3.0 \pm 5.0 & 6 \\ \text { M8 } & 6 & -1 & +9 & +3.0 \pm 6.7 & 9.5 \\ \text { HD 142096 } & 12 & +31 & 0 & +2.2 \pm 2.6 & 30 \\ \text { M16 } & 17 & +1 & +1 \text { and } & -4.5 \pm 12.5 & 6 \\ \text { W43 } & 31 & +1 & +24 & -2.0 \pm 21.0 & 6.5 \\ \text { IV-cloud } & 108 & +71 & -29 & -2.5 \pm 7.4 & 50 \\ \text { Cas A } & 112 & -2 & 0 & +0.2 \pm 0.7 & 21 \\ \text { 3C 123 } & 170 & -11 & +5 & +1.0 \pm 11.2 & 10 \\ \text { Dust cloud } & 174 & -14 & +6 & -4.7 \pm 4.5 & 26 \\ \text { Cloud C } & 238 & +38 & +6 & +4.5 \pm 5.3 & 23 \\ \text { CP 1133 } & 240 & +69 & 0 & -2.0 \pm 4.9 & 24 \\ \text { HD 147550 } & 350 & +25 & 0 & +7.0 \pm 11.0 & 13\end{array}$

a A negative sign indicates a field toward the observer.

density, which will be the value of the undisturbed "interstellar" magnetic field. Kerr (1969) has given a value of $0.7 \mathrm{~cm}^{-3}$ for the mean density in the region of 4 to $11 \mathrm{kpc}$ from the galactic center, whereas in the direction of Scorpius, Jenkins et al. (1969) find $2 \mathrm{~cm}^{-3}$ as the mean value from $L y-\alpha$ data. Considering these values as extremes I find from Figure 4 that the mean interstellar magnetic field is between 1 and $3 \mu \mathrm{G}$. Taking a value of $2 \mu \mathrm{G}$ I find the energy density of the field to be $1.6 \times 10^{-13} \mathrm{erg} \mathrm{cm}^{-3}$ and, if $I$ assume a radius of $15 \mathrm{kpc}$ and a thickness of $200 \mathrm{pc}$, I derive a total magnetic field energy of $6 \times 10^{53} \mathrm{erg}$ in the Galaxy.

\section{Pulsar Data}

The rotation measure is given by $\mathrm{RM}=0.81 \int n_{e} B_{\|} \mathrm{d} l$. The dispersion of the pulsed radiation yields a 'dispersion measure' $\mathrm{DM}=\int n_{e} \mathrm{~d} l$. When both quantities are measured, we readily obtain $B_{\|}$. Clearly this is the mean field in the line of sight weighted by the electron density. 
TABLE V

Mean fields in the direction of pulsars

\begin{tabular}{|c|c|c|c|c|c|c|}
\hline Pulsar & $l^{\text {II }}$ & $b^{\mathrm{II}}$ & 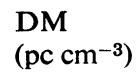 & $\begin{array}{l}\mathrm{RM} \\
\left(\mathrm{rad} \mathrm{m}^{-2}\right)\end{array}$ & References & $\begin{array}{l}\text { Field } \\
(\mu \mathrm{G})\end{array}$ \\
\hline AP $2015+28$ & 68.1 & -4.0 & 14.2 & -30 & a & +2.0 \\
\hline CP 0328 & 145.0 & -1.2 & 26.8 & $\begin{array}{l}-95 \\
+63\end{array}$ & $\begin{array}{l}a \\
d\end{array}$ & $\begin{array}{l}+3.5 \\
+2.8\end{array}$ \\
\hline $\begin{array}{r}\text { NP } 0532 \\
(\text { Crab) }\end{array}$ & 184.5 & -5.8 & 56.9 & -25 & c & +0.6 \\
\hline NP 0527 & 183.8 & -6.9 & 49.3 & +36 & $\mathrm{~d}$ & +0.9 \\
\hline CP 0950 & 228.9 & +43.7 & 2.98 & $<4$ & $\mathrm{~b}$ & $<0.2$ \\
\hline PSR 0833-45 & 263.5 & -2.8 & 63 & $\begin{array}{l}+42 \\
+33\end{array}$ & $\begin{array}{l}e \\
f\end{array}$ & $\begin{array}{l}-0.8 \\
-0.7\end{array}$ \\
\hline CP 0808 & 139.9 & +31.6 & 5.8 & -11 & $\mathrm{~g}$ & +2.3 \\
\hline
\end{tabular}

a Rotation measures are negative for a field away from the observer. However, in the Zeeman experiment a negative sign is taken to mean a field toward the observer.

References: $a=$ Smith, 1968b; $b=$ Smith, 1968a $; c=R M$ from Morris and Berge, 1964; $d=$ Staelin and Reifenstein, 1969; $\mathrm{e}=$ Radhakrishnan, 1969; $\mathrm{f}=$ Ekers et al., 1969; $\mathrm{g}=$ Smith, F. G. (private communication).

Table $\mathrm{V}$ shows data for seven pulsars together with the reference for the rotation measures. Values of DM may be found in many papers (e.g., Taylor, 1969; Davidson and Terzian, 1969; Bridle and Venugopal, 1969; Davies, 1969). The average value for the mean field in Table $\mathrm{V}$ is about $1.6 \mu \mathrm{G}$. This agrees well with the Zeeman data discussed before, although the statistics are not yet good.

The interpretation of the Zeeman data in the previous section suggested that high fields can only occur in relatively dense $\mathrm{HI}$ clouds. CP 0328 has two dense absorbing clouds in front of it (Gordon et al., 1969), and Hjellming et al. (1969) have derived a mean density of $8 \mathrm{~cm}^{-3}$ in these clouds, assuming a kinetic temperature of $50 \mathrm{~K}$ and a distance to the pulsar of $1 \mathrm{kpc}$. According to Figure 4 the line-of-sight field component is about $4 \mu \mathrm{G}$ in clouds of this density. The pulsar data alone give approximately $3 \mu \mathrm{G}$, but this value is an average weighted by the electron density. A combination of a path length of $50 \mathrm{pc}$ in cold clouds, having $n_{e}=4.4 \times 10^{-2} \mathrm{~cm}^{-3}$ and a field of about $4( \pm 2) \mu \mathrm{G}$, with a pathlength of $\approx 1 \mathrm{kpc}$ of intercloud medium, having a field of $2 \mu \mathrm{G}$ and $n_{e}=2.6 \times 10^{-2} \mathrm{~cm}^{-3}$ gives RM-values of 8 and $42 \mathrm{rad} \mathrm{m}^{-2}$ respectively, which compares well with the observed total value of $63 \mathrm{rad} \mathrm{m}^{-2}$ (Staelin and Reifenstein, 1969). For this pulsar the intercloud medium dominates in producing the rotation measure. Radhakrishnan (1969) has pointed out that in the direction of PSR 0833-45 there is no dense $\mathrm{HI}$ cloud. The rotation measure is $+42 \mathrm{rad} \mathrm{m}^{-2}$, which must then be due to the intercloud medium alone unless a significant amount is due to the Vela-X supernova remnant and the associated HiI regions.

In the case of the Crab Nebula pulsar the picture is somewhat complicated. The RM data indicate a mean field toward the source, the Zeeman data show a cloud with a field in the opposite direction. The line of sight to the source probably goes through 
parts of two spiral arms and the $\mathrm{HI}_{\mathrm{I}}$ absorption data indicate at least four to six absorbing clouds in the line of sight. The Zeeman splitting gave $3.5 \mu \mathrm{G}$ in one of these, but the pulsar data give a very low average field of $0.6 \mu \mathrm{G}$. Clearly, field reversals must be occurring, or the contribution to the RM from the Crab Nebula itself dominates the total RM.

\section{Some Problems in the Interpretation of Faraday Rotation Measurements}

In the distribution on the sky of the Faraday rotation measures (RM) several large irregularities are found. While it is true that the largest RM-values are found within $10^{\circ}$ from the galactic plane, it seems hard to justify the statement that the RM-values decrease with increasing latitude for $|b|>10^{\circ}$. For example, a radio source that is usually ignored is $3 \mathrm{C} 287$ at $b^{\mathrm{II}}=81^{\circ}$ with $\mathrm{RM}=+67 \mathrm{rad} \mathrm{m}^{-2}$. Admitting that some sources have a large intrinsic RM weakens all subsequent discussion! Other exceptions may be found among the five new RM-values obtained by Berge and Seielstad (1969). One is CTA 21 (or PKS 0316+16) with a RM of $+240 \mathrm{rad} \mathrm{m}^{-2}$ which lies within $3^{\circ}$ of the radio sources PKS $0300+16$ and PKS $0307+16$; the latter two, according to Gardner et al. (1969a), have RM-values of -17 and $-15 \mathrm{rad} \mathrm{m}^{-2}$. In this context

TABLE VI

Orientation of $\mathrm{HI}$ clouds with respect to magnetic field

$l^{\text {II }} \quad b^{\text {II }}$

Axial ratio

Position Direction of

angle magnetic field

Difference

Intermediate velocity clouds

$\begin{array}{llllllr}\mathrm{A} & 136 & +54.5 & 2: 1 & 105^{\circ} & 90^{\circ} \mathrm{O}, \mathrm{T} & 15^{\circ} \\ \mathrm{B}^{\mathrm{a}} & 146.7 & +39.5 & \geqslant 3: 1 & 100^{\circ} & 95^{\circ} \mathrm{T} & 5^{\circ} \\ \mathrm{C} & 104 & +69 & 2: 1 & 55^{\circ} & 60^{\circ} \mathrm{T} & 5^{\circ} \\ \mathrm{D}(1) & 86.5 & +59.5 & 2: 1 & 50^{\circ} & 60^{\circ} \mathrm{T} & 10^{\circ} \\ \quad(2) & 89.0 & +56.5 & 5: 1 & 50^{\circ} & 60^{\circ} \mathrm{T} & 10^{\circ} \\ \mathrm{E} & 102 & +36 & 2: 1 & 90^{\circ}(+10) & 70^{\circ} \mathrm{T}\left(98^{\circ} \mathrm{O}\right) & 20^{\circ} \\ \text { RII } & 136 & +51.5 & 3: 1 & 110^{\circ} & 90^{\circ} \mathrm{T} & 20^{\circ} \\ \text { RI } & 148 & +38.0 & 3: 1 & 110^{\circ} & 100^{\circ} \mathrm{T} & 10^{\circ}\end{array}$

High velocity clouds

$\begin{array}{lrllrlr}\text { MI } & 160 & +66 & 4: 1 & 102^{\circ} & 105^{\circ} \mathbf{T} & 3^{\circ} \\ \text { MII } & 185 & +65 & 5: 1 & 120^{\circ} & 130^{\circ} \mathbf{T} & 10^{\circ} \\ \text { CI } & 90 & +45 & \text { Large } & 50^{\circ} & 65^{\circ} \mathbf{T} & 15^{\circ} \\ \text { CIII } & 120 & +52 & 3: 1 & 80^{\circ} & 80^{\circ} \mathrm{O}, \mathbf{T} & 0^{\circ} \\ \text { Sm } & 43 & -15 & 3: 1 & 130^{\circ} & 150^{\circ} \mathrm{O}, \mathrm{T} & 20^{\circ} \\ \text { Sp } & 50 & -80 & 3: 1 & 135^{\circ} & 130^{\circ} \mathrm{O}\left(160^{\circ} \mathrm{T}\right) & 5^{\circ} \\ \text { A } & 150 & +35 & 7: 1 & 40^{\circ} & 100^{\circ} \mathbf{T} & 60^{\circ} \\ \text { B } & 168 & +38.5 & 2: 1 & 140^{\circ} & 110^{\circ} \mathrm{T} & 30^{\circ} \\ \text { ACI } & 183 & -12 & 3: 1 & 120^{\circ} & 45^{\circ} \mathrm{T} & 75^{\circ} \\ \text { ACII } & 192 & -22 & 7: 1 & 140^{\circ} & 50^{\circ} \mathrm{T} & 90^{\circ}\end{array}$

a H I data on this cloud are of poor quality. 


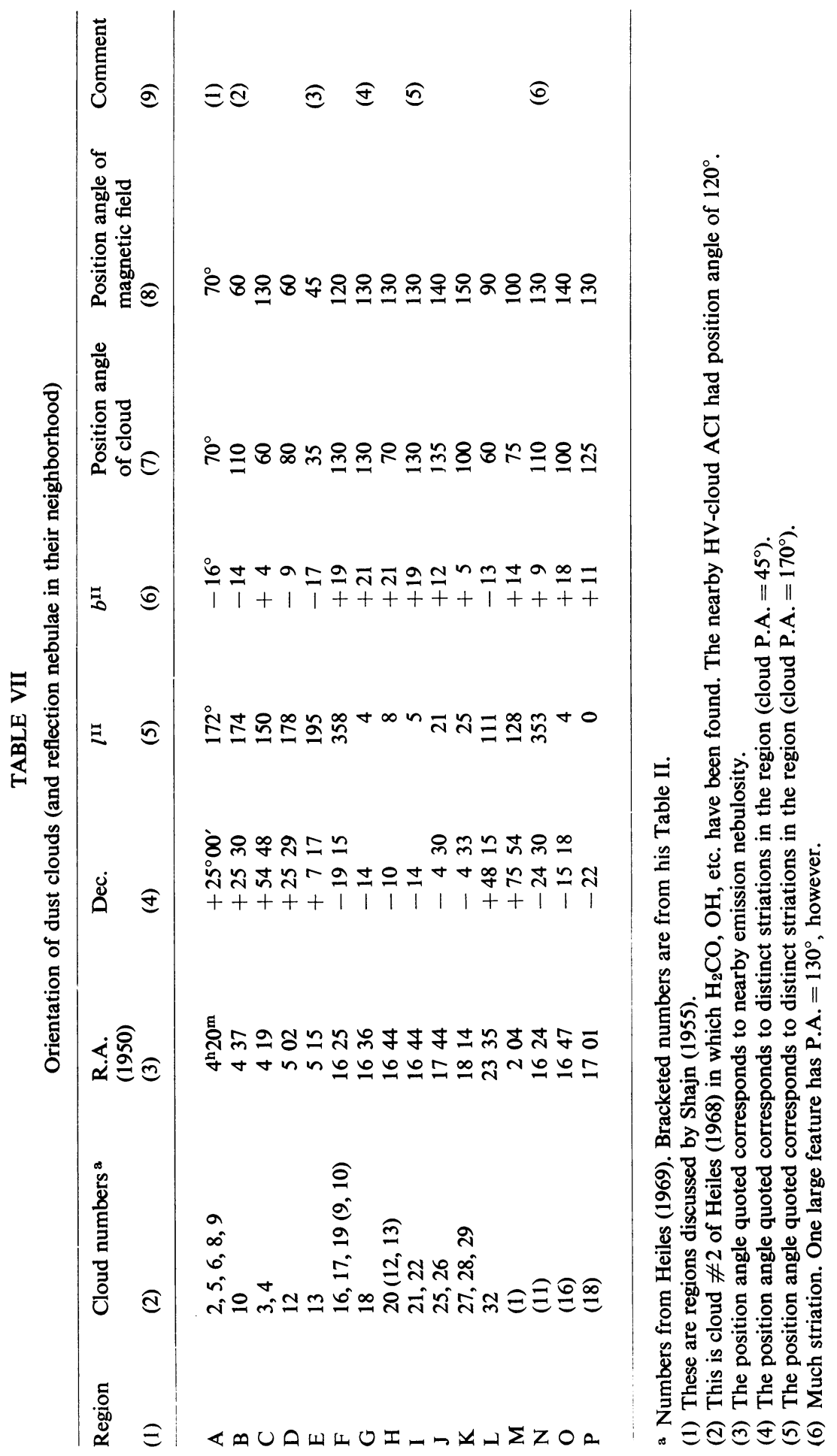


reference is made to the suggestion by Gardner, Morris, and Whiteoak of the existence of 'magnetic loops' and to the suggestion by Bologna et al. (1969, see also Bologna et al., 1965) of the presence of small-scale depolarizing structures. Davies (1968) has also proposed small-scale depolarization but this analysis has been criticized by Gardner et al. (1969b).

Sofue et al. (1969) have suggested that the RM of an extragalactic radio source depends on the redshift. From this they derive an intergalactic magnetic field of $10^{-9} \mathrm{G}$. I have plotted recently obtained RM-values in the diagrams of Sofue et al., and the result is not an overwhelming support for their case, but the plots warrant further investigation.

There is obviously much to be done in the interpretation of RM-data. We must not be too disappointed if a simple picture of our galactic field pattern is not derived.
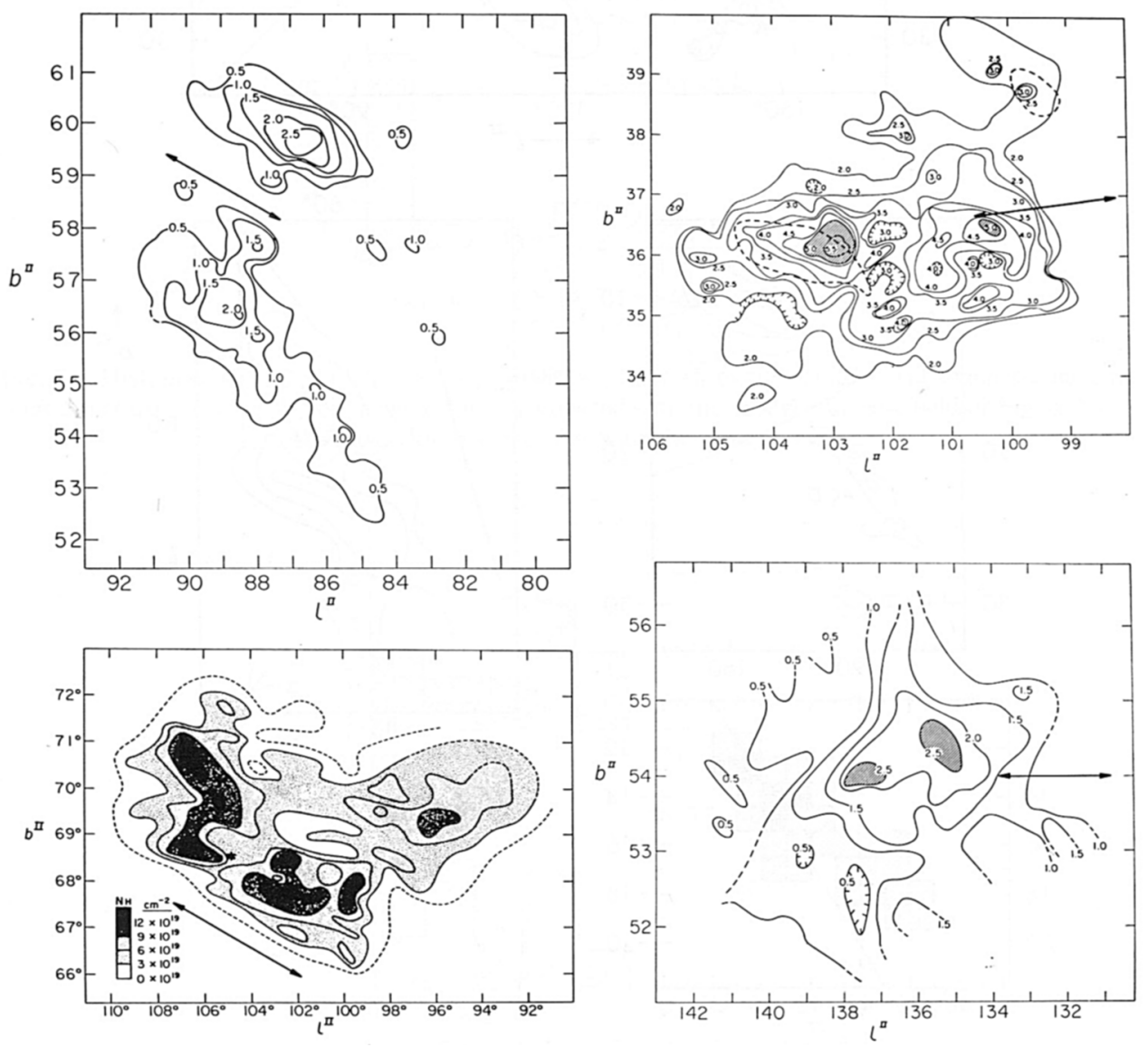

Fig. 5. Four intermediate velocity clouds of neutral hydrogen with heavy arrows representing the magnetic field vectors in that direction from the model (or data) in Figure 1. Contours are integrated hydrogen density in units of $10^{20}$ atom $\mathrm{cm}^{-2}$ except for lower left cloud. 


\section{Evidence for Cloud Elongation Parallel to the Magnetic Field}

In the study of a cloud at high latitudes it was noted (Verschuur, 1969e) that the cloud is elongated in the direction of the magnetic field, as found from the polarization of background radiation. To see whether such an alignment is a more common phenom-
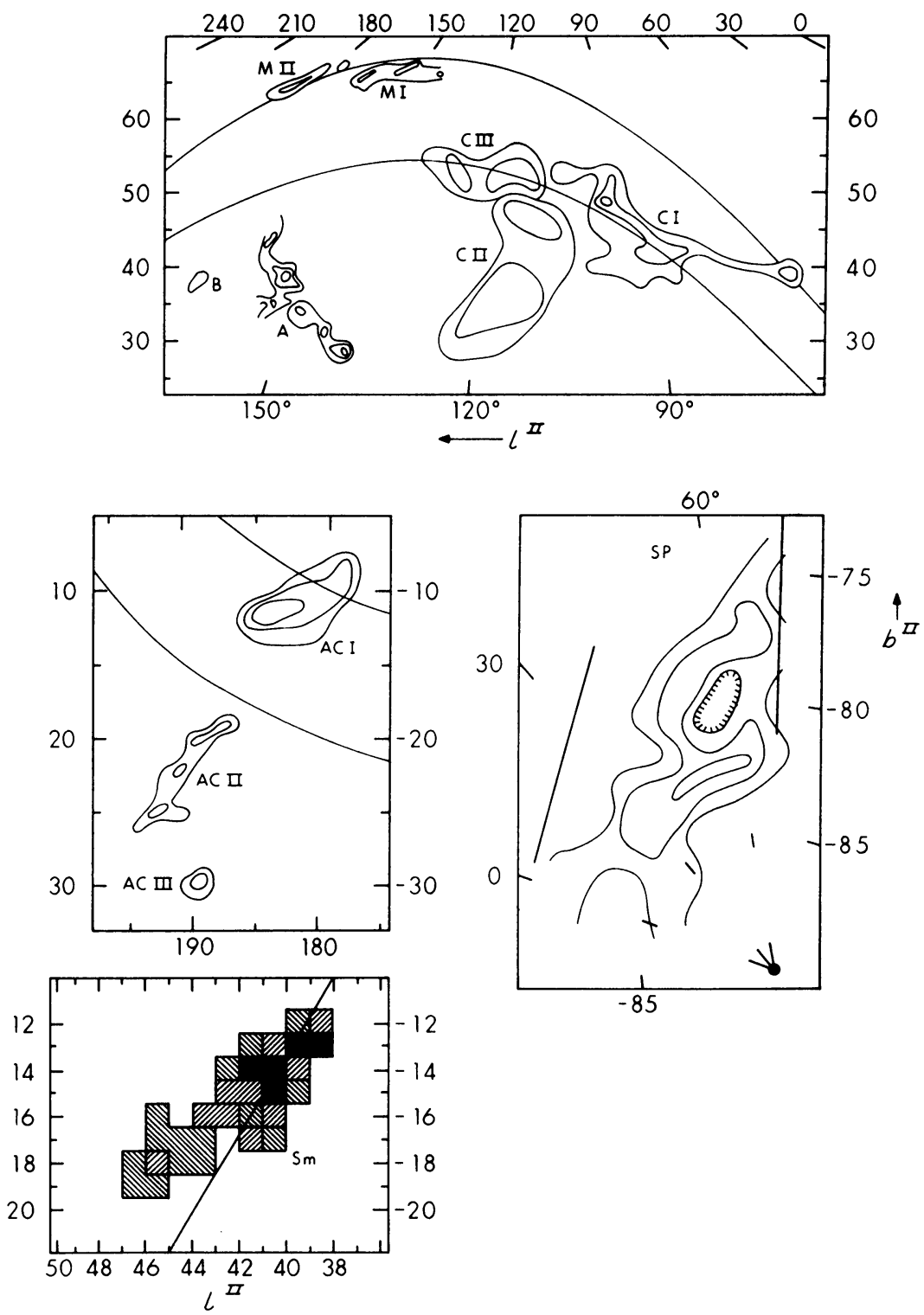

Fig. 6. The magnetic field lines in the region of the high velocity clouds taken from Hulsbosch (1968) and Smith (1963). Heavy lines indicate the field vectors taken from Figure 1. 
enon I have collected in Table VI data on eight intermediate velocity (IV) clouds $\left(|\mathrm{V}|<70 \mathrm{~km} \mathrm{sec}^{-1}\right)$ and on ten high-velocity (HV) clouds. The data are from Verschuur (1969e, f, g), from Rickard (1969), from Hulsbosch (1968), and from Smith (1963). Column 6 contains the position angle of the magnetic field read from Figures
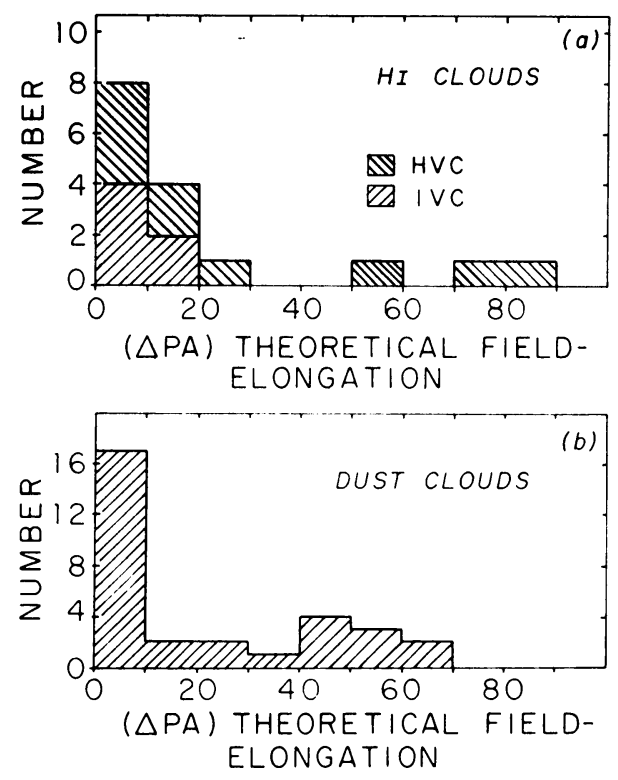

Fig. 7. Histograms of the difference in position angle of elongated clouds and magnetic field in their direction. - (a) Neutral hydrogen clouds compared with the model magnetic field of Figure 1b. -

(b) Dust clouds compared with the same model.

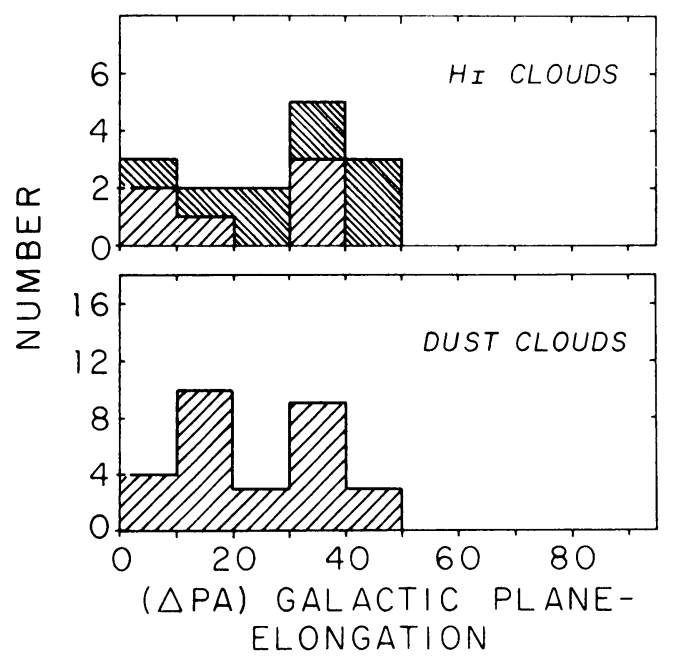

Fig. 8. Histogram of the difference between cloud axes and the galactic plane. 
$1 \mathrm{a}$ or $1 \mathrm{~b}$. Figure 5 shows maps of 5 of the IV-clouds, and in Figure 6 some HV-clouds are presented. All the IV-clouds appear elongated in the direction of the (helical) magnetic field and 6 out of the $10 \mathrm{HV}$-clouds do the same. The HV-clouds as a group do not give the impression of parallel alignment. It is clear that high resolution observations of the HV-clouds are essential to decide whether small-scale features exist within them, which might be indicative of the presence of magnetic fields. This, in turn, would argue strongly for these clouds to be inside our Galaxy.

Shajn (1955) has already noted that a number of dust clouds were elongated parallel to the optical polarization vectors of stars in their neighbourhood. We have briefly followed this up by taking the list of dust clouds given by Heiles (1968) and comparing the elongations, if any, of these clouds with the direction of the magnetic field read of Figures $1 \mathrm{a}$ or $1 \mathrm{~b}$. Table VII shows the data used. The position angle of the cloud (column 7) was obtained by inspecting the Palomar Sky Survey Plates. The histograms of the difference in the expected field direction and cloud position angle (column 7 minus column 8) is also shown in Figure 7. In Figure 8 we have plotted the histogram of position angles of both the hydrogen and dust clouds compared to the galactic plane to illustrate that the clouds are more closely aligned parallel to the field direction than the galactic plane.

It is worth reviving Meaburn's (1965) model for HV-clouds in which he noted that they occur near the spurs of galactic continuum emission. This is still true for the HV-clouds discovered since then, as well as for the IV-clouds. If, as Mathewson suggests, the spurs are the result of explosions in the plane injecting relativistic electrons along the field lines then perhaps these explosions have injected $\mathrm{HI}$ clouds along these lines as well. This, however, does not explain the predominance of negative velocities.

\section{Summary}

The local magnetic field appears to be helical in shape. This is consistent with optical polarization data, with the existence of spurs of galactic continuum emission and with their polarization. A basic longitudinal field may exist beyond the helix, which may be regarded as a local field pocket in which the net optical polarization is produced. Many such field pockets probably exist in the Galaxy. Spurs of continuum emission are the result of injection of non-thermal electrons into parts of the helical field. Zeemaneffect data show that the magnetic field in neutral hydrogen clouds is proportional to their density and that the basic interstellar field has been amplified in gravitationally contracting clouds. Extrapolation to a mean intercloud density suggests a mean interstellar field of $2 \pm 1 \mu \mathrm{G}$. This is partially supported by the fact that 10 other clouds have fields less than $10 \mu \mathrm{G}$. Pulsar data show that the mean field, weighted by electron density, is also of the order of a few $\mu \mathrm{G}$. It is not yet possible to derive the field strength in the local helical field region unambiguously.

Many interstellar clouds, both $\mathrm{HI}_{\mathrm{I}}$ and dust, are elongated parallel to the helical field lines. The overall motion of these clouds may also be determined by the field. 


\section{References}

Appenzeller, I.: 1968, Astrophys. J. 151, 907.

Behr, A.: 1959, Veröff. Univ.-Sternw. Göttingen, No. 126.

Berge, G. L. and Seielstad, G. A.: 1969, Astrophys. J. 157, 35.

Bingham, R. G.: 1967, Monthly Notices Roy. Astron. Soc. 137, 157.

Bologna, J. M., McClain, E. F., Rose W. K., and Sloanaker, R. M.: 1965, Astrophys. J. 142, 106.

Bologna, J. M., McClain, E. F., and Sloanaker, R. M.: 1969, Astrophys. J. 156, 815.

Bridle, A. H. and Venugopal, V. R.: 1969, Nature 224, 545.

Clark, B. G.: 1965, Astrophys. J. 142, 1398.

Clube, S. V. M.: 1968, Observatory 88, 243.

Davidson, K. and Terzian, Y.: 1969, Astron. J. 74, 849.

Davies, R. D.: 1968, Nature 218, 435.

Davies, R. D.: 1969, Nature 223, 355.

Davies, R. D., Booth, R. S., and Wilson, A. J.: 1968, Nature 220, 1207.

Ekers, R. R., Lequeux, J., Moffet, A. T., and Seielstad, G. A.: 1969, Astrophys. J. Lett. 156, L21.

Gardner, F. F. and Davies, R. D.: 1966, Australian J. Phys. 19, 129.

Gardner, F. F., Morris, D., and Whiteoak, J. B.: 1969a, Australian J. Phys. 22, 79.

Gardner, F. F., Morris, D., and Whiteoak, J. B.: 1969b, Australian J. Phys. 22, 813.

Gardner, F. F. and Whiteoak, J. B.: 1969, Australian J. Phys. 22, 107.

Gordon, C. P., Gordon, K. J., and Shalloway, A. M.: 1969, Nature 222, 129.

Hall, J. S.: 1958, Publ. U.S. Naval Observ., 2nd series, 17, No. VI.

Heiles, C.: 1968, Astrophys. J. 151, 919 (\# 2 list).

Heiles, C.: 1969, Astrophys. J. 156, 493.

Hiltner, W. A.: 1956, Astrophys. J. Suppl. $2,389$.

Hjellming, R. M., Gordon, C. P., and Gordon, K. J.: 1969, Astron. Astrophys. 2, 202.

Hulsbosch, A. N. M.: 1968, Bull. Astron. Inst. Netherl. $20,33$.

Jenkins, E. B., Morton, D. C., and Matilsky, T. A.: 1969, Astrophys. J. 158, 943.

Kerr, F. J.: 1969, Ann. Rev. Astron. Astrophys. 7, 39.

Krzeminski, W. and Serkowski, K.: 1967, Astrophys. J. 147, 988.

Mathewson, D. S.: 1968, Astrophys. J. Lett. 153, L47.

Mathewson, D. S.: 1969, Proc. Astron. Soc. Australia 1, 209.

Mathewson, D. S. and Nicholls, D. C.: 1968, Astrophys. J. Lett. 154, L11.

Meaburn, J.: 1965, Nature 207, 179.

Morris, D. and Berge, G. L.: 1964, Astrophys. J. 139, 1388.

Radhakrishnan, V.: 1969, Proc. Astron. Soc. Australia 1, 6.

Rickard, J. J.: 1969, preprint.

Seymour, P. A. H.: 1969, Monthly Notices Roy. Astron. Soc. 142, 33.

Shajn, G. A.: 1955, Astron. Zh. 32, 381.

Shuter, W. L. H. and Verschuur, G. L.: 1964, Monthly Notices Roy. Astron. Soc. 127, 387.

Smith, F. G.: 1968a, Nature 220, 892.

Smith, F. G.: 1968b, Nature 218, 325.

Smith, G. P.: 1963, Bull. Astron. Inst. Netherl. 17, 203.

Sofue, Y., Fujimoto, M., and Kawabata, K.: 1969, Publ. Astron. Soc. Japan 20, 388.

Staelin, D. H. and Reifenstein, E. C.: 1969, Astrophys. J. Lett. submitted for publication.

Taylor, J. H.: 1969, Astrophys. Lett. 3, 205.

Van de Hulst, H. C.: 1967, Ann. Rev. Astron. Astrophys. 5, 179.

Verschuur, G. L.: 1968, Phys. Rev. Lett. 121, 775.

Verschuur, G. L.: 1969a, Astrophys. J. 156, 861.

Verschuur, G. L.: 1969b, Nature 223, 140.

Verschuur, G. L.: 1969c, Astrophys. J. Sept. 1970.

Verschuur, G. L.: 1969d, Astrophys. J. Lett. 155, L155.

Verschuur, G. L.: 1969e, Astron. Astrophys. 1, 473.

Verschuur, G. L.: 1969f, Astron. Astrophys. 3, 77.

Verschuur, G. L.: $1969 \mathrm{~g}$, in preparation. 